\title{
The Cosmology Gallery: Unity through diversity in a vast and awe-inspiring universe.
}

\author{
John Goldsmith \\ Gravity Discovery Centre, \\ PO Box 313, Gingin, Western Australia 6503 \\ email: John.Goldsmith.MSc@gmail.com
}

\begin{abstract}
Scientists, artists, religious and cultural leaders have come together to create the Cosmology Gallery at the Gravity Discovery Centre (GDC) located $70 \mathrm{~km}$ north of Perth, Western Australia. The Cosmology Gallery exhibitions include the multicultural cosmology artworks, Celestial Visions astronomical photography exhibition and the Timeline of the Universe. The multicultural cosmology artworks are new artworks inspired by Australian Indigenous, Christian, Buddhist, Islamic, Hindu, scientific and technological perspectives of the universe. The Celestial Visions exhibition features astronomical events above famous landmarks, including Stonehenge and the Pyramids. The AUD 400,000+ project was funded by Lotterywest, Western Australia and the Cosmology Gallery was officially opened in July 2008 by the Premier of Western Australia.
\end{abstract}

Keywords. cosmology, exhibitions, astronomical photography, celestial visions, multicultural cosmology artworks, art, Gravity Discovery Centre, Australian Indigenous, Aboriginal

\section{Introduction: the cosmology garden}

The Cosmology Gallery is a place where modern cosmology meets multicultural creation stories, expressed through the art of the great world religious-cultural groups. Its motto is Unity through Diversity in a Vast and Awe Inspiring Universe. The unity is the universal human yearning to understand our place in the universe, which is expressed through the rich diversity of traditional cosmology and modern scientific cosmology. The opening of the Cosmology Gallery, in July 2008, was featured in Nature $\dagger$. The Gallery is one of the main facilities of the Gravity Discovery Centre, located 70 kilometres north of Perth, Western Australia

\section{A collaborative multicultural cosmology project}

The multicultural cosmology artwork project and the Cosmology Gallery is based on extensive collaboration and involvement of the community, religious and cultural leaders, scientists, photographers and artists. Concepts for the Cosmology Gallery were developed through several public lectures and forums, which helped to generate ideas and input for the Cosmology Gallery. These public forums also demonstrate the enormous public interest in the Cosmology Gallery concept. Public presentations included: (1)Einstein Buddha and Modern Cosmology (February 2006) Prof. David Blair; (2) Finding our Place in the Universe (January 2007) Prof. David Blair; (3) Are Science and Religion Incompatible? (February 2007) Prof. David Blair, Ajahn Brahm, Abbot of Bodhiyana 
Buddhist Monastery \& Abbott Placid Spearit; (4) What Happened Before the Big Bang? (August 2007) Sir Roger Penrose; (5) Awesome Universe: Cosmology in Art, Science and Culture (March 2008) Prof David Blair \& John Goldsmith.

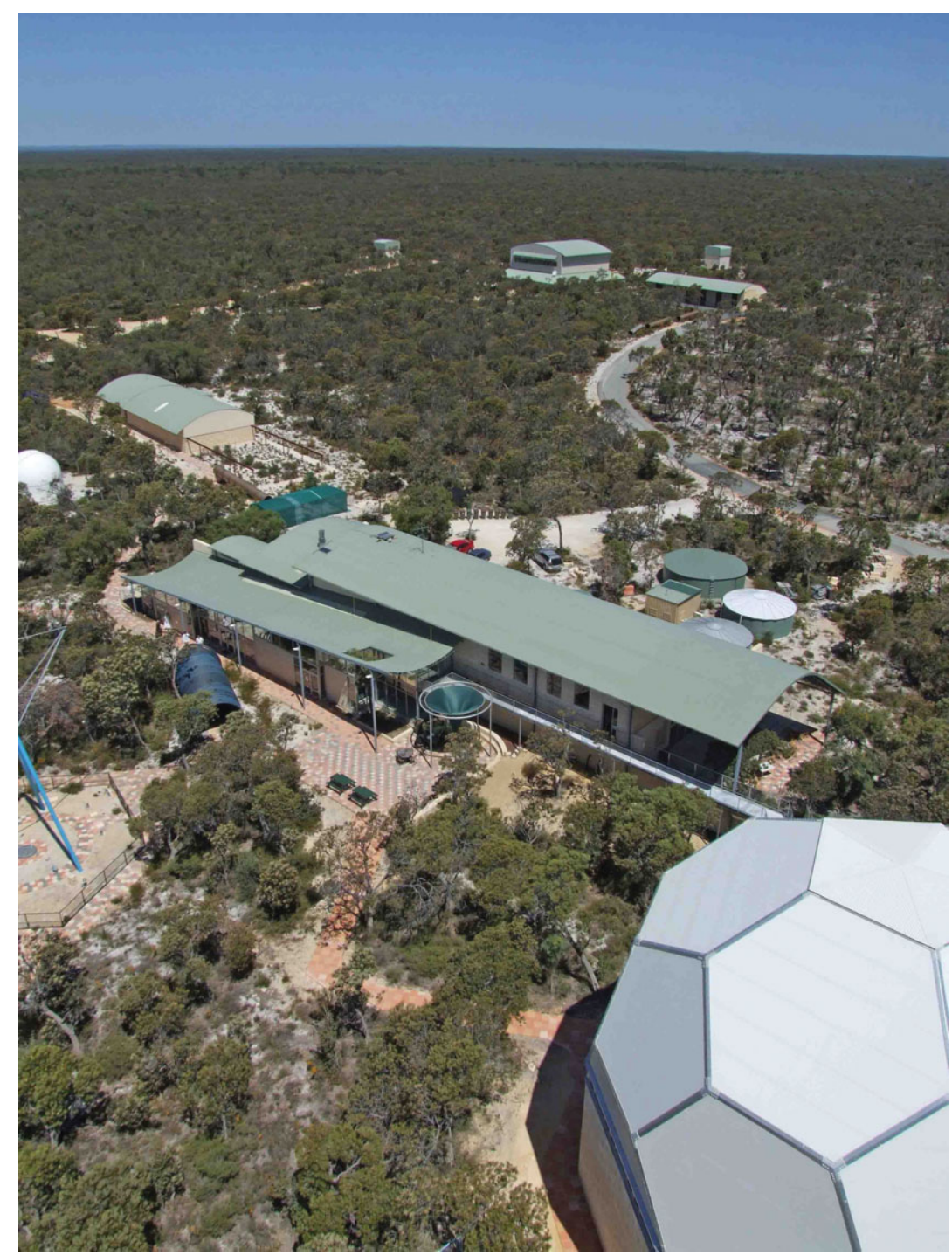

Figure 1. The Gravity Discovery Centre (as viewed from the United Group Leaning Tower of Gingin). (Lower right): Cosmology Gallery. Central building: Gravity Discovery Centre, (Upper left): Gingin Observatory, (Far upper left): Zadko Telescope. )Top centre): Australian International Gravitational Research Centre.

It is estimated that over 800 people have attended the above public forums. In July 2007, artists, scientists, cultural, religious and community leaders gathered together to explore the subject of cosmology and the creation of the universe. This fascinating forum provided many interesting and informative perspectives about cosmology, including perspectives from Indigenous, Christian, Buddhist, Hindu, Jewish and Islamic traditions. Artists were short-listed, based on community groups putting forward their suggestions and expression of interest from the arts community. This process resulted in the final 
selection of artists, who were engaged to participate in the cosmology project. During the development and creation of artworks, many artists continued their interaction with their community groups, thereby gaining important guidance for their artwork.

The act of creating artworks has been documented by several video productions, in which artists describe their ideas and how they have approached the subject of cosmology. The documentaries have been developed by Mr George Karpathakis and students from Edith Cowan University, in conjunction with the Film \& Television Institute (Western Australia). The videos are now presented in the Cosmology Gallery.

In August 2007, the Cosmology Gallery was privileged to host Sir Roger Penrose, English mathematician and cosmologist. Sir Roger is one of the Patrons of the Cosmology Gallery and his famous Penrose tiling is featured at the gallery. The Penrose tiling is generated by the combination of two geometric shapes, which combine to create nonrepeating patterns. During his visit to Western Australia, Sir Roger also gave a public presentation What Happened Before the Big Bang? (August 2007).

\section{The Cosmology Gallery exhibits}

The three main exhibits of the Cosmology Gallery include the multicultural cosmology artworks, the Celestial Visions astronomical photography exhibition and the Timeline of the Universe exhibit (Gravity Discovery Centre 2008).

The following artwork descriptions have been adapted from the gallery exhibit.

Multicultural Cosmology Artworks present new interpretations of cosmology and creation stories by Australian Indigenous, Christian, Buddhist, Islamic, Hindu \& Scientific perspectives.

The Cosmology Gallery presents two artworks inspired by Australian Indigenous cosmology. The first is entitled Bibbullmun Religion by Toogarr Morrison. The exhibit comprises nine canvas artworks, $(170 \times 230 \mathrm{~cm} \mathrm{\&} 8 \times 53 \times 41 \mathrm{~cm})$. Bibbullmun Religion provides a representation of Indigenous cultural cosmology knowledge, from the South West of Western Australia. As the Morrison Family sat around the camp fire in the bush where we lived, after finishing the evening meal, our attention was tuned into what our father was saying. He talked of the stars, and as he pointed upwards, our heads followed as our eyes focused, the surrounding foliage of the trees seemed to form a circle that represented a huge lens of which we could observe the universe. The story of the stars, play an important role in a belief of our existence on mother earth. We were told of Woor-jall-luk and her camp fire burning bright near her kaunt (hut). The story of how she made our camping grounds on earth and how the Milky Way came to be a weaving net that held the stars together. The Dreaming of the Bibbullmun history and religion encompassed a great understanding of ritual that was endorsed into our understanding of our environment from child hood to adulthood as we looked through the circle of firelight to the light of our cosmology.

The second Indigenous inspired artwork is entitled Garnkiny Ngarrangkarni (2007) by artist Mabel Juli. The artwork is natural ochre and pigments on canvas, $120 \times 120$ $\mathrm{cm}$. Mabel Juli is from Warmun Community, Turkey Creek in the far north of Western Australia. The painting shows a highly simplified representation of the crescent moon and star, and relates the story from Yarring country in Darrajayin (Springvale Station) which lies south of Warmun.

In the Ngarrangkarni (Dreaming) Garnkiny, the moon was a man. One day Garnkiny came back from hunting kangaroo and saw a girl sitting with her mother. She was very beautiful with long black hair and he fell in love with her instantly. This girl was Daawool, the black headed snake. However, it was taboo for him to marry her. The old people asked 

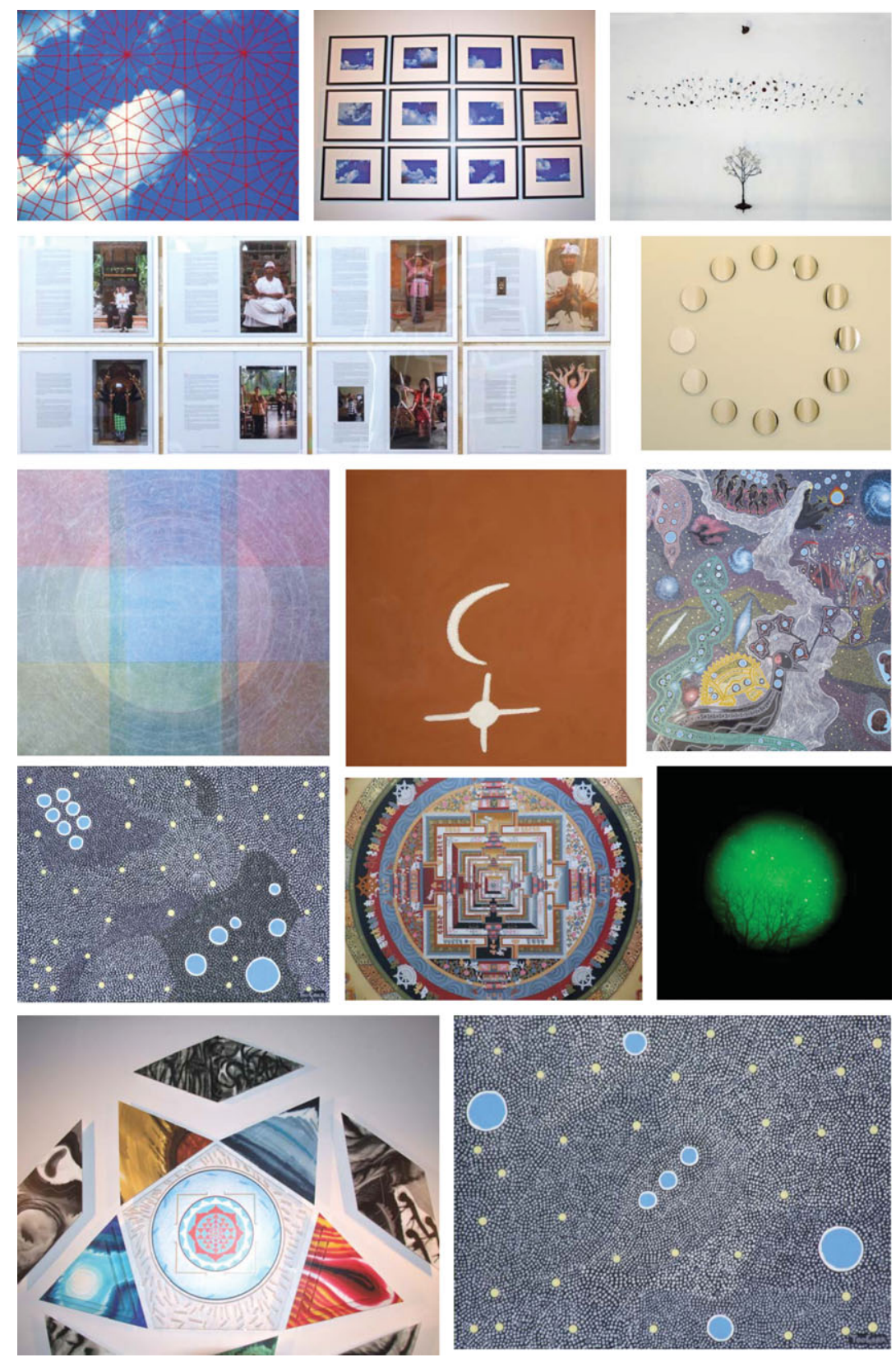

Figure 2. Multicultural Cosmology Artworks. Exhibits at the Cosmology Gallery include artworks inspired by cultural creations stories by Australian Indigenous (South Western Australia and Kimberley), Christian, Buddhist, Hindu, Islamic artists and artists inspired by scientific and technological perspectives.

him, Who do you want for your wife?. He told them dawyan dawyan (that one, that one) pointing to Daawool, but they said, No, she's your mother-in-law, you must marry one of these promised girls, these nyawana, Daawool's daughters. Again they asked him who he wanted to marry and he said once more dawyan dawyan. The people then told him, You must go away! He strode off angrily and walked some distance before he sat and turned 
into a hill. He cursed the people, telling them that they were going to die, but that he would always live. As the Moon, he comes back to life every month. The wardal (stars) are Daawool's daughters.

Scientific and technological perspectives are represented in two artworks Night Vision (2006) by artist Perdita Phillips and Lunar Circle: Figure $C$ by artist Marion Borgelt. Night Vision comprises four digital prints on paper, $4 \times 86 \times 86 \mathrm{~cm}$. This work arose from an intensive field project in the Kimberley of Western Australia which sought to examine the intersection of art and science in the field. The images were made using a night vision scope. Like many forms of technology it extends the boundaries of our perception outward into the void. It features details of a campsite, a compass and film stock, the Southern Cross, and a sideways glance at the shadow of a still, tropical night. Lunar Circle: Figure $C$ (2007) by artist Marion Borgelt, is about the heartbeat of our solar system using the subject of the Moon as it waxes and wanes through its monthly cycle. From new Moon to full Moon and back to new Moon, this sculpture implies an eternal rhythm of movement whose sequence is divided into 12 movements using gradually folding forms to identify each movement. Accordingly, the full Moon is represented by the full, flattened circle and the new Moon is represented by the most closed form. Every universal body is in constant motion that is governed by an individual 'heartbeat' and every heartbeat has a pulse or rhythm. The rhythm of the heartbeat can be measured mathematically and each mathematical sequence becomes a system for understanding the relationship of the human being to the greater universe and to Time itself.

Our Moon, as it orbits the Earth, seemingly changes appearance due to its position in relation to the earth and sun. From the position of the Moon in the sky man has devised a system to measure time in terms of months and years. This gives us a sense of motion, sequence and order in the universal scheme of things; it gives us a way to measure our own lives and what we might achieve and how long things take. We can structure our objectives according to the time required for achieving them by using our time wisely and judiciously.

Buddhist inspired artworks are represented in two exhibits. Bardo of Becoming (2008) by artist Eveline Kotai, who is a founding member (under the guidance of Geshe Jampel Senge) of the Tashi Choeling Buddhist Centre in Whitegum Valley, Western Australia. The artwork (oil on Belgian linen, $183 \times 198 \mathrm{~cm}$ ) is a representation of cause and effect, the centre of all Buddhist philosophy. Buddhists speak of time as beginning-less and see the universe as something in flux, passing in and out of existence and parallel to an infinite number of other universes doing the same thing. As well as a model of universal origins and destruction, Buddhist cosmology also functions as a model of the mind, with its thoughts coming into existence based on preceding thoughts, and being transformed into other thoughts and other states. A deep understanding of impermanence, the interconnectedness of all phenomena and the practice of compassion form the Buddhist principles for mind transformation.

The second Buddhist inspired artwork is Tibetan Mandala (date unknown) by artist Dun Zhu, an acclaimed Thangka artist, born in Lundup, Dzong County, Tibet in 1961 and now lives in Lhasa. This work is a Kalachakra mandala, which represents time and the universe, physical and mental aspects of ourselves, and aspects of Buddhist practice. Usually the mandala is surrounded by spheres representing Earth, water, fire, wind, space and wisdom. Kalachakra meditation is a form of meditation in which one concentrates on visualisation of the complete intricate detail of such a mandala. The exhibit is acrylic on silk, $105 \times 105 \mathrm{~cm}$.

The Islamic inspired cosmology artwork Pieces of Sky (2008) is by artist David Sequeira (assisted by Chris Malcolm). The geometric patterns that form the basis of Islamic art 
are at once both highly decorative and profoundly metaphoric. They represent in symbol what is thought to be the essential structure of the universe. Islamic patterns are based upon the division of the circle with each element inter-relating with the next to form a unified design. Such patterns can be understood as expressing the notion of the cosmos in the sense that the substance of the pattern is conceived as an organised, unified whole. The artwork comprises $1250 \times 40 \mathrm{~cm}$ framed digital photos with polyester string sewn through the prints.

The Christian inspired artwork Dust to Dust (2008) by Therese Howard, is a sculpture cast in bronze, patinated and cold painted with silver. It is inspired by Genesis 3: 17-19 (King James Version) for dust thou art, and unto dust shalt thou return. This installation derives its narrative structure from the theory of an ever expanding universe, one created from a single infinitely dense point that expands to eventually create stars and their planets, galaxies, constellations, dark matter and black holes. The single event that began this chain reaction is most commonly called the Big Bang. There is also speculation that the universe will continue to expand until it eventually collapses back into itself -the Big Crunch- and then the cycle of inflation and contraction will continue.

Hindu inspired cosmology artworks are represented in two major works. Everyone (2008) by artists Rodney Glick, Christopher Hill, Sohan Hayes, comprises eight digital prints, $96 \times 120 \mathrm{~cm}$ (framed). Everyone features the stories and beliefs of Balinese Hinduism. The artwork is a series of digital prints and creation myth interviews highlighting ordinary people as Gods. The sitters are anonymous, suggesting a universality in both their stories and photographic portraits. Each individual's interview and portrait is presented graphically as if they are separate pages from a very large book, in many volumes, containing every person in the world. The suite of prints shows a range of different age groups, intended to represent a fictional family group. Each digital print has both the portrait of the 'family member' and some text with their own view on how the world began and their place in the cosmos. The sitters were interviewed in either Balinese or Indonesian and the interviews were then edited and translated into English. The portraits are of adults and children adopting poses that suggest a divine presence. Each sitter was photographed in several choreographed movements and then all poses were digitally manipulated into one image.

Traveling to Bali and working closely with a distinct local community has been a cultural exchange that has bought the artists many memorable experiences and enhanced their understanding of Balinese religious beliefs. By documenting cultural stories through text and digital imagery, the artists have begun to appreciate the diverse creation theories within a community, which in turn helps everyone to reflect and contemplate the beliefs held within their own society.

The second Hindu inspired artwork is Sunyata (2008) by Audrey Satar. The concept of Sunyata is derived from the Sanskrit term for nothingness or emptiness. This artwork explores creation narratives within Hindu cosmology and spirituality, relating to the making of the world and the beginning of consciousness. Sunyata is a multi-media installation that links the notion of phenomenal existence through the properties of light and movement. This installation incorporates three layers or levels of the creation story unfolding in a sequence, beginning with the Sri Chakram at the centre of the artwork that represents the universe. The second layer is dedicated to the five elements -Earth, Water, Fire, Air and Akasha, the Sanskrit word for aether. The third layer is dedicated to the creation of all Life on Earth and the cycle of life that moves from Grass to Worm-like, to Trees to Animals to Birds to Snakes to Stones to Humans to Spirits and Heavenly Beings. 


\section{Celestial Visions astronomical photography exhibition}

The Celestial Visions photographic exhibition presents beautiful images of the cosmos together with famous places, world heritage sites and landscapes of great scenic beauty. The exhibition is a unique collection and has developed from over 20 years of astronomical photography. The exhibition explores the profound connection between the celestial realm and our own biological, cultural and earthly experience and comprises four main collections, 'Sun and Moon', 'Celestial Wilderness', 'Ancient Skies' and 'Celestial Cities'. Diverse astronomical events are featured including major comets, meteor showers, eclipses of the Moon and Sun, views of our own Galaxy, the Milky Way, and many other astronomical events.

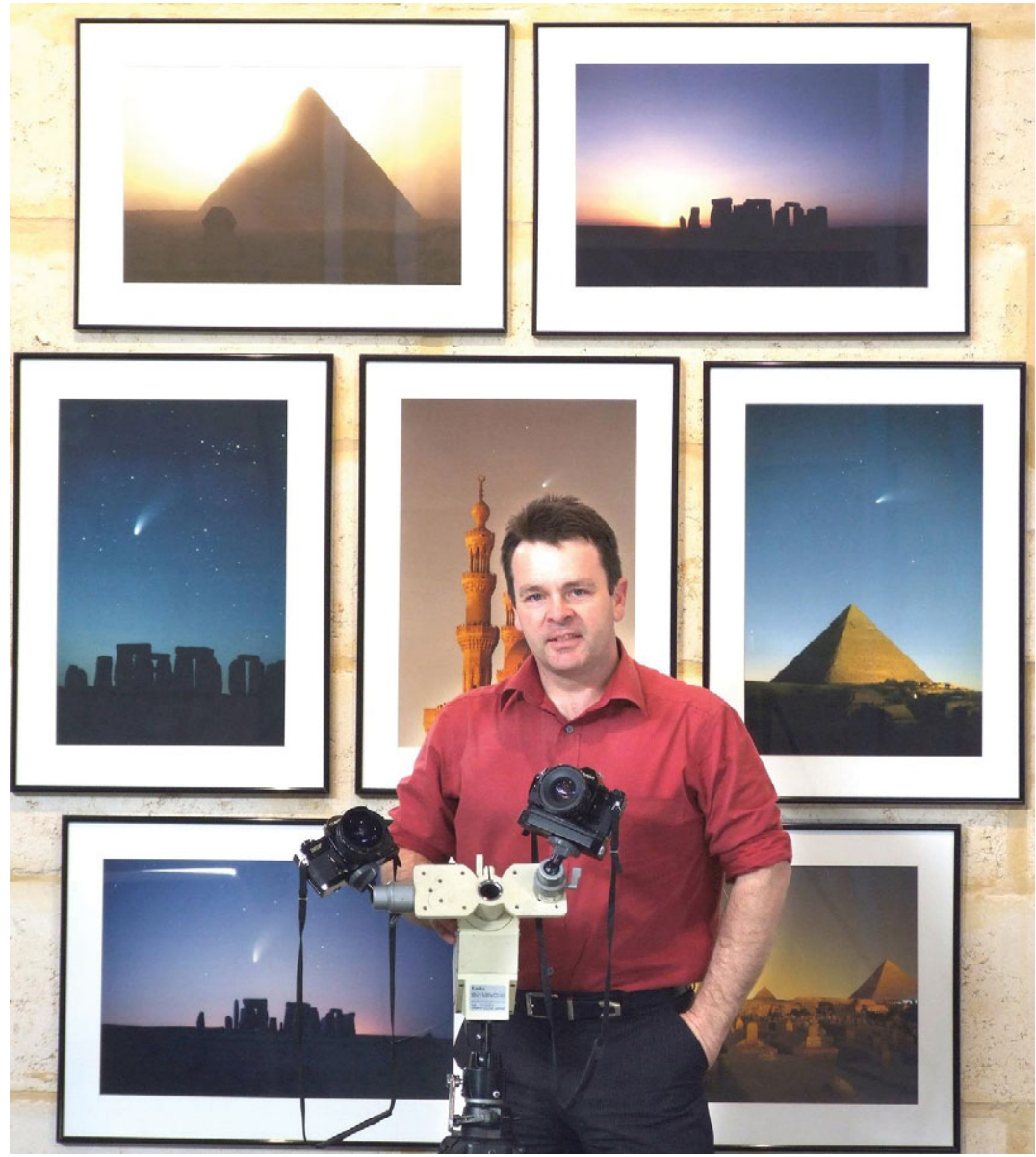

Figure 3. Celestial Visions astronomical photography exhibition presents astronomical events with natural and cultural landscapes in four main collections, 'Sun \& Moon', 'Celestial Wilderness', 'Ancient Skies' and 'Celestial Cities'. World Heritage locations such as the pyramids and Stonhenge are featured.

'Sun and Moon' features images of sunrise, the annular solar eclipse of 1991 and a total eclipse of the Moon. The Sun is a profound symbol of unity for all civilisations throughout the millennia, while the Moon is symbolic of cyclic renewal in nature.

'Celestial Wilderness' reveals brilliant stars glowing in magnificently dark skies, above wild and remote landscapes. This series of images features the stars as they appear above 
famous and beautiful Western Australian landscapes including the world heritage Bungle Bungles, the Pinnacles (Nambung National Park) and Wolfe Creek Meteorite Crater (all located in Western Australia).

'Ancient Skies' explores the fascinating links between ancient cultures and the stars. The collection includes rare images of Comet Hale-Bopp, which was discovered in 1995 and then developed into a very prominent comet in 1997. The orbit of the comet indicates that it last appeared over 4000 years ago and is not expected to return for another couple of thousand years. The co-incidence of the comet's appearance during ancient times inspired my documentary photography project in which I set out to record the comet appearing above two of the most famous ancient monuments, the Pyramids of Giza and Stonehenge. Ancient monuments such as the Pyramids demonstrate precise alignments which are astronomically derived. Celestial inspiration is also apparent in Islamic cultures. The symbolic crescent moon adorns the top of minaret towers. Ancient stone circles such as Stonehenge have long bee $\mathrm{n}$ associated with astronomical alignments including the solstice. We can gain new insights into human development by learning about ancient cultures and their relation to the stars and the cosmos.

'Celestial Cities' includes contemporary images of the urban and city environment related to astronomical events, including the spectacular appearance of Comet McNaught in January 2007. The series also includes special astronomical events which contrast with the city and urban views of the night sky. The contrast emphasises the impact of civilisation on our view of the cosmos, now partially obscured by light pollution.

The Celestial Visions exhibition enables viewers to explore the many connections between the astronomical world and human culture. These connections are revealed in cultural creation stories of the cosmos, ancient monumental structures inspired by the celestial, modern scientific endeavours to understand the universe.

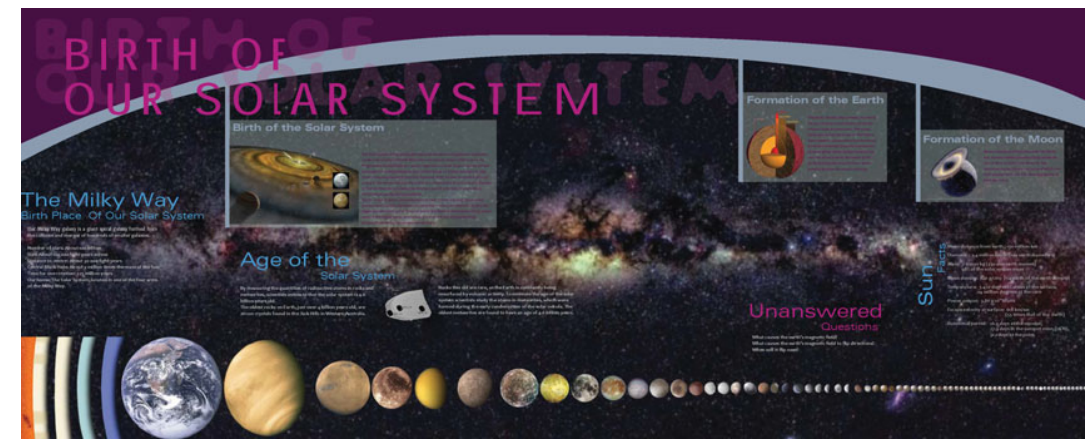

Figure 4. The Timeline of the Universe exhibit is a 60 metre exhibit presenting the story of the original, evolution and future of the universe, featured at the Cosmology Gallery.

\section{Timeline of the Universe}

The Timeline of the Universe presents the history of the universe along a 60 metre display, viewed from the spiral walkway within the Cosmology Gallery. The exhibition was conceived and developed by Geoff Cody and presents the astonishing scientific story of the origin and evolution of the universe in photographs, text and exhibits. The timeline explores the history of the universe, the Big Bang, the synthesis of atoms, the birth of galaxies and stars, the supernova that led to the solar system, the evolution of the Earth, the ascent of life, the rise of civilisations and the future of our planet. The exhibit uses 
local Western Australian examples to illustrate the timeline including Western Australian geological and fossil exhibits, loaned by the Museum of Western Australia.

\section{The Gravity Discovery Centre}

The Cosmology Gallery is part of the Gravity Discovery Centre, which is a public education facility dedicated to exploring the big questions of life and the universe, innovation and creativity, science and spirituality The centre is an exciting mix of innovative projects including a 1 metre robotic telescope, a large public astronomy centre, a 1 kilometre scale model of the solar system, a major gravitational wave research facility and the Leaning Tower of Gingin - a modern structure which emphasises the links with Galileo and his famous gravity experiments at the Leaning Tower of Pisa.

The Gravity Discovery Centre features galleries and exhibits which present the story of gravity research, a lecture theatre, shop front and café. Guided tours are provided in addition to educational modules for primary and secondary students.

The Gingin Observatory (Southern Cross Cosmos Centre) is the public astronomy centre at the Gravity Discovery Centre. The observatory includes several large telescopes which are used for practical astronomy viewing evenings.

The United Group Leaning Tower of Gingin is a 13 story high structure designed with a 15 degree lean. Opened to the public in 2008, the Leaning Tower of Gingin is a unique attraction. The tower is inspired by Galileo and the Leaning Tower of Pisa, Italy. Visitors to the Leaning Tower of Gingin can test and validate Galileo's experiments and Sir Isaac Newton's Laws of Motion.

The Zadko Telescope is a one metre Cassergrain robotic telescope, designed for the automated search of near earth asteroids and transitory astronomical events, such as gamma ray bursts. The telescope was installed in 2008 and is being developed to be operated online with collaborators in Chile and France, for use by school children and researchers. The telescope is managed by The University of Western Australia.

\section{Future Directions}

The Cosmology Gallery now provides a permanent collection of artworks to provide an enduring benefit to our community. It is hoped that the Cosmology Gallery will help to inspire and encourage greater understanding of cultural views of the universe and our place in it. We hope the Cosmology Gallery collection will grow and develop over time, through future projects, sponsorship and donations.

\section{Acknowledgements}

The Gravity Discovery Centre Foundation (Inc) acknowledges and thanks the many individuals, companies, artists and community/cultural based groups and all funders, who have contributed or participated in the development of the gallery. Special thanks goes to the State Government of Western Australia, Department of Culture and the Arts, Lotterywest, WA Government Regional Infrastructure Programme, Office of Multicultural Interests, Public Education Endowment Trust, Film and Television Institute of Western Australia, The University of Western Australia, Edith Cowan University, participating artists and members of the Cosmology Gallery Development Group. 